Pacific

Journal of

Mathematics

QUANTUM STIEFEL MANIFOLD AND DOUBLE COSETS OF QUANTUM UNITARY GROUP

G.B. Podkolzin And L.I. Vainerman

Volume 188 No. 1

March 1999 


\title{
QUANTUM STIEFEL MANIFOLD AND DOUBLE COSETS OF QUANTUM UNITARY GROUP
}

\author{
G.B. Podkolzin and L.I. Vainerman
}

Dedicated to the memory of Professor Yuri L. Daletskii

\begin{abstract}
We study the homogeneous space of the quantum group $U_{q}(n)$ related to the subgroup $U_{q}(n-m)(m<n)$, classify its irreducible representations and get a formula for its invariant integral. We also study the double cosets $U_{q}(n-$ $m) \backslash U_{q}(n) / U_{q}(n-m)$ and the hypergroup structure associated with them.
\end{abstract}

\section{Introduction.}

If a group $G$ acts on a set $S$ transitively on the right, then one can view $S$ as $G / K$, where $K$ is a subgroup of $G$. Thus a function on $S$ can be considered as a function on $G$, invariant with respect to the right shifts by elements of $K$. It is especially interesting to consider bi-invariant functions on $G$ since they can be identified with the functions on the set of $G$-orbits in $S$. If $G$ is a locally compact group and $K$ is its compact subgroup with Haar measures $\mu_{G}$ and $\mu_{K}$ respectively, then the set $B \subset L_{1}\left(G, \mu_{G}\right)$ of all bi-invariant functions is an algebra with respect to the convolution and has a natural hypergroup structure related to generalized translation operators (see the survey $[\mathbf{2 3}]$ and the references given there):

$$
R^{h} f(g)=\int_{K} f(g k h) d \mu_{K}(k)=\Delta(f)(g, h), \quad g, h \in G .
$$

If the subalgebra $B$ is commutative ( or, equivalently, the coproduct $\Delta$ is cocommutative) then $(G, K)$ forms a Gel'fand pair [6]. In many cases the characters of $B$ are well known special functions of mathematical physics. This explains the importance of the notion of a Gel'fand pair.

The case of a noncommutative subalgebra $B$ has been studied in a number of papers. In particular, a pair $(S O(n), S O(m))$ was considered in [8], [22]. It is known that the homogeneous space $S O(n) / S O(m)$ can be regarded as a Stiefel manifold $S^{n, n-m}$. The infinitesimal object for the corresponding hypergroup structure was investigated in $[\mathbf{1 5}],[\mathbf{1 6}]$.

A similar situation arises while considering functions on compact quantum groups [29] (see also [20]). Here, for a pair of compact quantum groups 
$\left(\mathcal{H}_{1}, \mathcal{H}_{2}\right)$ and a surjection $\pi: \mathcal{H}_{1} \rightarrow \mathcal{H}_{2}$, we also consider an algebra $H$ of biinvariant elements and define a coalgebra structure on it [4], [24], [25], [26]. In this situation we say that $H$ is endowed with a hypergroup structure. If the coalgebra is cocommutative, we call $\left(\mathcal{H}_{1}, \mathcal{H}_{2}\right)$ a Gel'fand pair [12], [24]. If, in addition to, the algebra is commutative, we call such a Gel'fand pair strict $[24],[25]$.

When algebra and coalgebra of bi-invariant elements are noncommutative, a corresponding hypergroup structure is more complicated. One of the simplest examples of such a situation is given by the pair $\mathcal{H}_{1}=U_{q}(n), \mathcal{H}_{2}=$ $U_{q}(n-m)$ (quantum unitary groups, $m<n$ ). For this pair we study a structure of a homogeneous space $S_{q}^{n, m}=U_{q}(n) / U_{q}(n-m)$ which is a quantum analogue of a Stiefel manifold. We give its description in terms of generators and relations of commutation, obtain a classification of its irreducible representations and a formula for an invariant integral on it. These results generalize the results obtained in [14], [17], [21], [28] for $m=1$. In this case $S_{q}^{n, 1}$ are quantum spheres. We also investigate the double cosets $U_{q}(n-m) \backslash U_{q}(n) / U_{q}(n-m)$ and the corresponding hypergroup structure. In a special case $m=1$ these and related questions were studied in [4], [7], [11], [12], [24], [25], [26].

We are grateful to Prof. Yu.A. Chapovsky, A.A. Kaljuzhnui, A.U. Klimyk and T.H. Koornwinder for many useful discussions. The second author is grateful to Prof. C. Anantharaman and J. Renault for their kind hospitality and support during his stay in Orleans, where this work was finished.

\section{Preliminaries.}

All modules, comodules, algebras, coalgebras, Hopf algebras [1], linear maps, homomophisms, tensor products are considered over the field $C$ of complex numbers. Algebras (coalgebras) are associative (coassociative) unital (counital).

2.1. Quantum group $G L_{q}(n, C)[5],[14],[18],[19]$.

Let a Hopf algebra $\mathcal{H}:=A\left(G L_{q}(n, C)\right)$ be generated by letters $t_{i j}(i, j \in$ $\{1, \ldots, n\}), \operatorname{det}^{-1}{ }_{q}$ satisfying the following relations of commutation $(q \in C)$ :

$$
\begin{gathered}
t_{i k} t_{j k}=q t_{j k} t_{i k}, \quad t_{k i} t_{k j}=q t_{k j} t_{k i}(i<j), \\
t_{i l} t_{j k}=t_{j k} t_{i l}(i<j, k<l), \\
t_{i k} t_{j l}-q t_{i l} t_{j k}=t_{j l} t_{i k}-q^{-1} t_{j k} t_{i l}(i<j, k<l) . \\
\operatorname{det}_{q}^{-1} t_{i j}=t_{i j} \operatorname{det}_{q}^{-1} .
\end{gathered}
$$


The coproduct $\Delta: H \rightarrow H \otimes H$ and counit $\varepsilon: H \rightarrow C$ act in the following way:

$$
\begin{gathered}
\Delta\left(t_{i j}\right)=\sum_{k} t_{i k} \otimes t_{k j}, \Delta\left(\operatorname{det}_{q}^{-1}\right)=\operatorname{det}_{q}^{-1} \otimes \operatorname{det}_{q}^{-1}, \\
\varepsilon\left(t_{i j}\right)=\delta_{i j}, \quad \varepsilon\left(\operatorname{det}_{q}^{-1}\right)=1 .
\end{gathered}
$$

The antipode $S$ is a homomorphism $S: H \rightarrow H$ such that:

$$
\begin{gathered}
S\left(t_{i j}\right)=(-q)^{i-j} \xi_{\hat{i}}^{\hat{j}} \operatorname{det}_{q}^{-1}, \\
S\left(\operatorname{det}_{q}^{-1}\right)=\operatorname{det}_{q},
\end{gathered}
$$

where

$$
\xi_{J}^{I}=\sum_{\tau \in P_{r}}(-q)^{l(\tau)} t_{i_{\tau(1)} j_{1}} \cdots t_{i_{\tau(r)} j_{r}}
$$

is the quantum minor determinant; $\hat{k}=(1, \cdots, k-1, k+1, \cdots, n)$, $\operatorname{det}_{q}=$ $\xi_{1, \cdots, n}^{1, \cdots, n} \quad I=\left(i_{1}, \cdots, i_{r}\right) ; \quad J=\left(j_{1}, \cdots, j_{r}\right), P_{r}$ is the permutation group of the set $(1, \cdots, r) ; l(\tau)=(\tau(1), \cdots, \tau(r))$ is the number of inversions in $\tau$.

The commutation relations (2), (3), (4) can be rewritten as

$$
\mathbf{R}(\mathbf{T} \otimes \mathbf{T})=(\mathbf{T} \otimes \mathbf{T}) \mathbf{R},
$$

where $\mathbf{T}:=\left(t_{i j}\right)_{i, j=1, \ldots, n}=\sum_{i, j} t_{i j} E_{i j} \in \operatorname{Mat}\left(n, A\left(G L_{q}(n, C)\right)\right), \mathbf{T} \otimes \mathbf{T}=$ $\sum_{i, j, k, l} t_{i j} t_{k l} E_{i j} \otimes E_{k l}, \mathbf{R} \in \operatorname{Mat}(n, C) \otimes \operatorname{Mat}(n, C)$ is a so-called constant $R$-matrix of type $A_{n-1}[\mathbf{9}],[\mathbf{1 8}]$ :

$$
\mathbf{R}=q \sum_{i} E_{i i} \otimes E_{i i}+\sum_{i \neq j} E_{j i} \otimes E_{i j}+\left(q-q^{-1}\right) \sum_{i<j} E_{i i} \otimes E_{j j}
$$

$E_{i j}$ are matrix units.

Remark 1. The quantum minor determinants satisfy the following relations:

$$
\Delta\left(\xi_{J}^{I}\right)=\sum_{\# K=r} \xi_{K}^{I} \otimes \xi_{J}^{K}, \quad \varepsilon\left(\xi_{J}^{I}\right)=\delta_{I J}
$$

2.2. Quantum universal enveloping algebra $U_{q}(g l(n, C))[5],[14]$, $[18]$.

Let $L_{n}$ be a free $Z$-module of rank $n$ with a canonical basis $\left(\varepsilon_{1}, \cdots, \varepsilon_{n}\right)$ : $L_{n}=\oplus_{k=1}^{n} Z \varepsilon_{k}$. We fix a symmetric bilinear form $L_{n} \times L_{n} \rightarrow Z$ defined by $\left\langle\varepsilon_{i}, \varepsilon_{j}\right\rangle=\delta_{i j}$. An element of $L_{n}$ will be called an integral weight.

The quantum universal enveloping algebra $U_{q}(g l(n, C))$ is a $C$-algebra defined by generators $e_{k}, f_{k} \quad(k \in\{1, \ldots, n\})$ and $q^{\lambda} \quad\left(\lambda \in L_{n}\right)$ and the following relations of commutation:

$$
\begin{gathered}
q^{\lambda} e_{k} q^{-\lambda}=q^{\left\langle\lambda, \varepsilon_{k}-\varepsilon_{k+1}\right\rangle} e_{k}, q^{\lambda} f_{k} q^{-\lambda}=q^{-\left\langle\lambda, \varepsilon_{k}-\varepsilon_{k+1}\right\rangle} f_{k}, \lambda \in L_{n}, k \in\{1, \ldots, n\} \\
e_{i} f_{j}-f_{j} e_{i}=\frac{\delta_{i j}\left(q^{\varepsilon_{i}-\varepsilon_{i+1}}-q^{-\varepsilon_{i}+\varepsilon_{i+1}}\right)}{q-q^{-1}}, \quad i, j \in\{1, \ldots, n\}
\end{gathered}
$$




$$
\begin{gathered}
e_{i}^{2} e_{j}-\left(q+q^{-1}\right) e_{i} e_{j} e_{i}+e_{j} e_{i}^{2}=0, f_{i}^{2} f_{j}-\left(q+q^{-1}\right) f_{i} f_{j} f_{i}+f_{j} f_{i}^{2}=0,|i-j|=1 \\
e_{i} e_{j}=e_{j} e_{i} ; f_{i} f_{j}=f_{j} f_{i} \quad|i-j|>1 \\
q^{0}=1, q^{\lambda} q^{\mu}=q^{\lambda+\mu} \quad \lambda, \mu \in L_{n} .
\end{gathered}
$$

This algebra has also a structure of a Hopf algebra with the following coproduct $\Delta$, counit $\varepsilon$, and antipode $S$ :

$$
\Delta\left(q^{\lambda}\right)=q^{\lambda} \otimes q^{\lambda}, \quad \varepsilon\left(q^{\lambda}\right)=1, \quad S\left(q^{\lambda}\right)=q^{\lambda}
$$

$$
\Delta\left(e_{k}\right)=e_{k} \otimes q^{-\left(\varepsilon_{k}-\varepsilon_{k+1}\right) / 2}+q^{\left(\varepsilon_{k}-\varepsilon_{k+1}\right) / 2} \otimes e_{k}, \quad \varepsilon\left(e_{k}\right)=0, S\left(e_{k}\right)=-q^{-1} e_{k}
$$$$
\Delta\left(f_{k}\right)=f_{k} \otimes q^{-\left(\varepsilon_{k}-\varepsilon_{k+1}\right) / 2}+q^{\left(\varepsilon_{k}-\varepsilon_{k+1}\right) / 2} \otimes f_{k}, \quad \varepsilon\left(f_{k}\right)=0, S\left(f_{k}\right)=-q f_{k} .
$$

Given two Hopf algebras $A$ and $H$ over $C$, we say that a $C$-bilinear form $\langle a, \phi\rangle: H \times A \rightarrow C$ is a pairing of Hopf algebras if it satisfies the following conditions:

$$
\begin{gathered}
\langle a, \phi \psi\rangle=\left\langle\Delta_{H}(a), \phi \otimes \psi\right\rangle, \quad\langle a, 1\rangle=\varepsilon_{H}(a) \\
\langle a b, \phi\rangle=\left\langle a \otimes b, \Delta_{A}(\phi)\right\rangle, \quad\langle 1, \phi\rangle=\varepsilon_{A}(\phi), \\
\left\langle S_{H}(a), \phi\right\rangle=\left\langle a, S_{A}(\phi)\right\rangle,
\end{gathered}
$$

for any $a, b \in H$ and $\phi, \psi \in A$.

The following proposition can be found in [5], [19]:

Proposition 1. There exists a unique pairing of Hopf algebras $U_{q}(g l(n, C))$ and $A\left(G L_{q}(n, C)\right)$ (briefly $G L_{q}(n, C)$ ), such that:

$$
\begin{gathered}
\left\langle q^{\lambda}, t_{i j}\right\rangle=\delta_{i j} q^{\left\langle\lambda, \varepsilon_{i}\right\rangle}\left\langle q^{\lambda}, \operatorname{det}_{q}^{m}\right\rangle=q^{m\left\langle\lambda, \varepsilon_{1}+\cdots+\varepsilon_{n}\right\rangle} \quad(m \in Z) \\
\left\langle e_{k}, t_{i j}\right\rangle=\delta_{i k} \delta_{j, k+1} ; \quad\left\langle f_{k}, t_{i j}\right\rangle=\delta_{i, k+1} \delta_{j k} \\
\left\langle e_{k}, \operatorname{det}_{q}^{m}\right\rangle=\left\langle f_{k}, \operatorname{det}_{q}^{m}\right\rangle=0(m \in Z) .
\end{gathered}
$$

Let $V$ be a right $G L_{q}(n, C)$-comodule (resp. left $G L_{q}(n, C)$-comodule) with a structure mapping $R_{G}: V \rightarrow V \otimes G L_{q}(n, C)$ (resp. $L_{G}: V \rightarrow$ $\left.G L_{q}(n, C) \otimes V\right)$, then $V$ has a left (resp. right) module structure over $U_{q}(g l(n, C))$ defined by

$$
a \cdot v=(\mathrm{id} \otimes a) \circ R_{G}(v) \quad\left(\text { resp. } v \cdot a=(a \otimes \mathrm{id}) \circ L_{G}(v)\right)
$$

$\forall a \in U_{q}(g l(n, C))$ and $v \in V$.

In particular, $G L_{q}(n, c)$ is a bimodule over $U_{q}(g l(n, C))$. The actions of the generators $q^{\lambda}, e_{k}, f_{k}$ are given by

$$
\begin{array}{rlrl}
q^{\lambda} t_{i j} & =t_{i j} q^{\left\langle\lambda, \varepsilon_{j}\right\rangle} ; & t_{i j} q^{\lambda}=t_{i j} q^{\left\langle\lambda, \varepsilon_{i}\right\rangle} \\
e_{k} t_{i j} & =t_{i, j-1} \delta_{j, k+1} ; & t_{i j} e_{k}=\delta_{i k} t_{i+1, j} ; \\
f_{k} t_{i j}=t_{i, j+1} \delta_{j k} ; & t_{i j} f_{k}=\delta_{i, k+1} t_{i-1 j} .
\end{array}
$$




\subsection{Quantum $G$-spaces and relative invariants [14].}

Let $G$ be a quantum group with a coordinate ring $A(G)$. Then a quantum space $X$ is called a quantum left $A(G)$-space if the coordinate ring $A(X)$ of $X$ has a structure of a left $A(G)$-comodule $L_{G}: A(X) \rightarrow A(G) \otimes A(X)$ such that $L_{G}$ is a $C$-algebra homomorphism. An element $\chi$ of $A(G)$ is called $a$ linear character of $G$ if

$$
\Delta(\chi)=\chi \otimes \chi, \quad \varepsilon(\chi)=1 .
$$

For a given linear character $\chi$ of $G$, an element $\phi$ of $A(X)$ is called a left relative invariant with character $\chi$ if $L_{G}(\phi)=\chi \otimes \phi$. The subspace of all left relative $G$-invariants in $A(X)$ with a character $\chi$ is denoted by

$$
(G \backslash X ; \chi)=\left(\phi \in A(X): L_{G}(\phi)=\chi \otimes \phi\right) .
$$

The notions of right $G$-space and right relative $G$-invariants are defined similarly. If $\chi=1$, the subalgebras of all right- and left- invariants are called quantum homogeneous spaces and denoted by $X \backslash G$ and $G / X$ respectively. In the similar way one can define a subalgebra of bi-invariants:

$$
G \backslash X / G=\left\{\phi \in A(X): R_{G}(\phi)=\phi \otimes 1, L_{G}(\phi)=1 \otimes \phi\right\} .
$$

\subsection{Corepresentations of $G L_{q}(n, C)[14]$.}

In what follows, we use an abbrevation $\xi_{J}=\xi_{j_{1} \ldots j_{r}}\left(\right.$ resp. $\left.\xi^{J}=\xi^{j_{1} \ldots j_{r}}\right)$ to refer to a quantum r-minor determinant $\xi_{j_{1} \ldots j_{r}}^{1 \ldots r}\left(\right.$ resp. $\left.\xi_{1 \ldots r}^{j_{1} \ldots j_{r}}\right)$ where $J=$ $\left(j_{1}<\cdots<j_{r}\right)$.

For each positive integer $r$ with $r \in\{1, \cdots n\}$, we define the fundamental weight $\Lambda_{r}$ by $\Lambda_{r}=\varepsilon_{1}+\cdots+\varepsilon_{r}$. Let $\lambda$ be an integral weight in $L_{n}$ in the form $\lambda=\Lambda_{m_{1}}+\cdots+\Lambda_{m_{l}}, \quad 0 \leq m_{l} \leq \cdots \leq m_{1} \leq n$. This condition is equivalent to stating that $\lambda$ is written as $\lambda=\lambda_{1} \varepsilon_{1}+\cdots+\lambda_{n} \varepsilon_{n}$ with $0 \leq \lambda_{n} \leq \cdots \leq \lambda_{1}$.

For such $\lambda$, let $T=\left(T_{r s} ; 1 \leq r \leq n, 1 \leq s \leq \lambda_{r}\right)$ be a family of elements in $(1, \ldots, n)$. If $T$ satisfies the conditions

$$
\begin{aligned}
& T_{r-1, s}<T_{r, s} \text { for } 1 \leq s \leq l, \quad 2 \leq r \leq m_{s}, \\
& T_{r, s-1} \leq T_{r, s} \text { for } 1 \leq r \leq n, \quad 2 \leq s \leq \lambda_{r},
\end{aligned}
$$

then $T$ is called a semi-standard tableau of shape $\lambda$ with labels in $(1, \ldots, n)$. We denote the totality of all semi-standard tableaus $T=\left(T_{r, s}\right)$ by $S \operatorname{STa} b_{n}(\lambda)$ and define the standard monomial $\xi_{T}$ indexed by $T$ as the product of quantum minor determinants

$$
\xi_{T}=\xi_{J_{1}} \ldots \xi_{J_{l}} \in G L_{q}(n, C),
$$

where $J_{s}=\left(T_{1, s}, \ldots, T_{m_{s}, s}\right)$ for $s \in\{1, \ldots l\}$.

Suppose now that $q$ is not a root of unity. 
Proposition 2. If $0 \leq \lambda_{n} \leq \cdots \leq \lambda_{1}$, then the standard monomials $\xi_{T}=$ $\xi_{J_{1}} \ldots \xi_{J_{l}}$ indexed by the semi-standard tableaus $T$ in $\operatorname{SSTab}_{n}(\lambda)$ form a $C$-basis for a right $G L_{q}(n, C)$-comodule denoted by $V^{R}(\lambda)$.

In a similar way one can construct a left irreducible $G L_{q}(n, C)$-comodule $V^{L}(\lambda)$. We say that an integral weight $\lambda$ in $L_{n}$ is dominant if $\lambda_{n} \leq \cdots \leq \lambda_{1}$.

Proposition 3. (1) If $\lambda$ is dominant, then the monomials $\left(\operatorname{det}_{q}\right)^{-m} \xi_{T}$ indexed by semi-standard tableaus $T$ in $S S T a b_{n}\left(\lambda+m\left(\varepsilon_{1}+\cdots+\varepsilon_{n}\right)\right)$ form a basis for the right $G L_{q}(n, C)$-comodule $V^{R}(\lambda)$ for any $m \in Z$ with $\lambda_{n} \geq-m$.

(2) Any finite-dimensional irreducible right (resp. left) $G L_{q}(n, C)-c o$ module is isomorphic to $V^{R}(\lambda)$ for some dominant integral weight $\lambda$ in $L_{n}$.

(3) Any finite-dimensional right and left $G L_{q}(n, C)$-comodule is completly reducible.

Proposition 4. The coordinate ring $A\left(C L_{q}(n, C)\right)$ is decomposed into the direct sum of irreducible two-sided $G L_{q}(n, C)$-comodules:

$$
A\left(G L_{q}(n, C)\right)=\oplus_{\lambda} W(\lambda)
$$

where the two-sided $G L_{q}(n, C)$-comodule $W(\lambda)$ is isomorphic to the tensor product of the left and right irreducible $G L_{q}(n, C)$-comodules $V^{L}(\lambda)$ and $V^{R}(\lambda)$ :

$$
W(\lambda) \sim V^{L}(\lambda) \otimes V^{R}(\lambda),
$$

and the summation runs over all dominant integral weights $\lambda$ in $L_{n}$.

\subsection{Invariant integral $[14],[29],[21],[27]$.}

Definition 1. A linear functional $\nu: G L_{q}(n, C) \rightarrow C$ is called right-invariant (resp. left-invariant) integral if

$$
(\nu \otimes \mathrm{id}) \circ \Delta(\phi)=\nu(\phi) \cdot 1 \quad(\text { resp. } \quad(\mathrm{id} \otimes \nu) \circ \Delta(\phi)=1 \cdot \nu(\phi))
$$

for all $\phi \in G L_{q}(n, C)$. A bi-invariant integral is called a Haar integral.

Proposition 5. There exists a unique Haar integral $h$ with $h(1)=1$ and it is the projection $\nu: \oplus_{\lambda} W(\lambda) \rightarrow W(0)$.

2.6. Quantum group $U_{q}(n)$ and quantum homogeneous space $U_{q}(n-$ 1) $\backslash U_{q}(n)[\mathbf{1 4}],[\mathbf{2 8}]$.

The definition of a Hopf *-algebra can be found in [20], [19]:

Definition 2. A Hopf algebra $\mathcal{H}$ is a Hopf *-algebra if it is equipped with a conjugate linear mapping $*: H \rightarrow H$, such that:

(1) $1^{*}=1 ;(\phi \psi)^{*}=\psi^{*} \phi^{*}$.

(2) $\varepsilon\left(\phi^{*}\right)=\overline{\varepsilon(\phi)} ; \Delta \circ *=(* \otimes *) \circ \Delta ; \quad(\forall \phi, \psi \in H)$.

(3) $* \circ *=\mathrm{id} ; * \circ S \circ * \circ S=\mathrm{id}$. 
Now we define the "compact real form" $U_{q}(n)$ of $G L_{q}(n, C)$ by introducing an involution in the Hopf algebra $G L_{q}(n, C)$, if $q$ is real, $q \notin\{-1,0,1\}$. The anti-homomorphism $*$ acts on $A\left(G L_{q}(n, C)\right)$ in the following way:

$$
t^{*}{ }_{i j}=S\left(t_{j i}\right) \quad \forall i, j \in\{1, \ldots, n\} \quad\left(\operatorname{det}_{q}^{-1}\right)^{*}=\operatorname{det}_{q} .
$$

One can check that $\mathbf{T}^{*} \mathbf{T}=\mathbf{T T}^{*}=1$, where $\mathbf{T}^{*}=\left(\left(t^{*}{ }_{j i}\right)_{i, j=1}^{n}\right)$, and that $A\left(U_{q}(n)\right)=A\left(\left(G L_{q}(n, C)\right), *\right)$ is a Hopf *-algebra.

Definition 3. The above Hopf $*$-algebra is the algebra of polynomials on the quantum unitary group $U_{q}(n)$. Sometimes we denote it briefly $U_{q}(n)$. The algebra of polynomials on the quantum group $S U_{q}(n)$ is a Hopf *-algebra specified by the condition $\operatorname{det}_{q}=1$ with the same $\Delta, \varepsilon, S, *$.

For $1 \leq m<n$ we define an epimorphism $\gamma_{m}: A\left(U_{q}(n)\right) \rightarrow A\left(U_{q}(n-m)\right)$ of Hopf $*$-algebras by:

$$
\gamma_{m}\left(t_{i j}\right)=s_{i j} \quad(1 \leq i, j \leq n-m)
$$

$$
\gamma_{m}\left(t_{k l}\right)=\delta_{k l} 1 \quad(k \text { or } l>n-m) ; \gamma_{m}\left(\operatorname{det}_{q}^{-1}\right)=\operatorname{det}_{q}^{-1} .
$$

Proposition 6. The algebra $U_{q}(n-1) \backslash U_{q}(n)$ is generated by $t_{n k}$ and $t_{n k}^{*}(1 \leq k \leq n)$, satisfying the following relations:

$$
\begin{gathered}
t_{n i} t_{n j}=q t_{n j} t_{n i}, q t_{n i}^{*} t_{n j}^{*}=t_{n j}^{*} t_{n i}^{*}(1 \leq i<j \leq n), \\
t_{n j}^{*} t_{n i}=q t_{n i} t_{n j}^{*}, 1 \leq i, j \leq n \\
t_{n k}^{*} t_{n k}=t_{n k} t_{n k}^{*}+\left(1-q^{2}\right) \sum_{l<k} t_{n l} t_{n l}^{*}(1 \leq k \leq n) \\
\sum_{k=1}^{n} t_{n k} t_{n k}^{*}=1 .
\end{gathered}
$$

The structure of the quantum homogeneous space $U_{q}(n) / U_{q}(n-1)$ is similar.

\subsection{Double cosets of quantum groups [4], [24], [25].}

Let $\mathcal{H}_{i}=\left(H_{i}, d_{i}, 1_{i}, \Delta_{i}, \varepsilon_{i}, S_{i}\right)(i=1,2)$ be two Hopf algebras, where $d_{i}$ is a product, $1_{i}$ is a unit, $\Delta_{i}$ is a coproduct, $\varepsilon_{i}$ is a counit, $S_{i}$ is an antipode in $H_{i}$. Let $\gamma: \mathcal{H}_{1} \rightarrow \mathcal{H}_{2}$ be an epimorphism of Hopf algebras, i.e. it is an algebra epimorphism such that $(\gamma \otimes \gamma) \circ \Delta_{1}=\Delta_{2} \circ \gamma, \varepsilon_{1}=\varepsilon_{2} \circ \gamma, \gamma \circ S_{i}=S_{2} \circ \gamma$.

With respect to the coactions

$$
\begin{gathered}
L_{H_{2}}=(\gamma \otimes \mathrm{id}) \circ \Delta_{1}, \quad L_{H_{2}}: H_{1} \rightarrow H_{2} \otimes H_{1} \\
\text { (resp., } \left.R_{H_{2}}=(\mathrm{id} \otimes \gamma) \circ \Delta_{1}, \quad R_{H_{2}}: H_{1} \rightarrow H_{1} \otimes H_{2}\right)
\end{gathered}
$$

$H_{1}$ is a left (resp., right) $H_{2}$-comodule. Define the sets $H_{2} \backslash H_{1}, H_{1} / H_{2}, H_{2} \backslash$ $H_{1} / H_{2}$ of left-, right- and bi-invariants in $H_{1}$ with respect to $H_{2}$ exactly as in 2.3 . 
Remark 2. a) Since $L_{H_{2}}$ and $R_{H_{2}}$ are homomorphisms, the above sets are unital subalgebras of $H_{1}$.

b) A straightforward verification shows that:

$$
\begin{gathered}
\Delta_{1}\left(H_{2} \backslash H_{1}\right) \subset H_{2} \backslash H_{1} \otimes H_{1}, \Delta_{1}\left(H_{1} / H_{2}\right) \subset H_{1} \otimes H_{1} / H_{2}, \\
\Delta_{1}\left(H_{2} \backslash H_{1} / H_{2}\right) \subset H_{2} \backslash H_{1} \otimes H_{1} / H_{2} .
\end{gathered}
$$

c) From $\Delta_{1} \circ S_{1}=\sigma \circ\left(S_{1} \otimes S_{1}\right) \circ \Delta_{1}$ (here $\left.\sigma(a \otimes b)=b \otimes a\right)$, one can deduce that

$$
\begin{gathered}
S_{1}\left(H_{2} \backslash H_{1}\right) \subset H_{2} \backslash H_{1}, S_{1}\left(H_{1} / H_{2}\right) \subset H_{1} / H_{2}, \\
S_{1}\left(H_{2} \backslash H_{1} / H_{2}\right) \subset H_{2} \backslash H_{1} / H_{2} .
\end{gathered}
$$

Let $\nu_{2}$ be an invariant integral on $\mathcal{H}_{2}$ (it exists if $\mathcal{H}_{2}$ is associated with a compact quantum group [29]). Introduce a new coproduct on $H_{2} \backslash H_{1} / H_{2}$ :

$$
\Delta:=\left(\mathrm{id} \otimes \nu_{2} \circ \gamma \otimes \mathrm{id}\right) \circ\left(\Delta_{1} \otimes \mathrm{id}\right) \circ \Delta_{1} .
$$

This is a generalization of (1) for Hopf algebras case.

We denote the restrictions of $\varepsilon_{1}, S_{1}$, to $H_{2} \backslash H_{1} / H_{2}$ by the same letters.

Proposition 7. Let a mapping $\Delta$ be defined by (11). Then:

a) $\Delta$ maps $H_{2} \backslash H_{1} / H_{2}$ into $H_{2} \backslash H_{1} / H_{2} \otimes H_{2} \backslash H_{1} / H_{2}$;

b) $\Delta$ is coassociative, i.e.

$$
(\mathrm{id} \otimes \Delta) \circ \Delta=(\Delta \otimes \mathrm{id}) \circ \Delta
$$

c) $\varepsilon_{1}$ is a counit on $H_{2} \backslash H_{1} / H_{2}$ with respect to $\Delta$ :

$$
\left(\varepsilon_{1} \otimes \mathrm{id}\right) \circ \Delta=\left(\mathrm{id} \otimes \varepsilon_{1}\right) \circ \Delta=\mathrm{id} ;
$$

d) if $\nu_{1}$ is a left- (resp., right-) invariant integral on $H_{1}$, then its restriction to $H_{2} \backslash H_{1} / H_{2}$ is left- (resp., right-) invariant with respect to $\Delta$ :

$$
\begin{gathered}
\left(\nu_{1} \otimes \mathrm{id}\right) \circ \Delta(h)=\nu_{1}(h) 1_{1} \\
\left(\text { resp. }, \quad\left(\mathrm{id} \otimes \nu_{1}\right) \circ \Delta(h)=\nu_{1}(h) 1_{1}\right) ;
\end{gathered}
$$

e) the following relation holds:

$$
\Delta \circ S_{1}=\sigma \circ\left(S_{1} \otimes S_{1}\right) \circ \Delta
$$

f) if both $\mathcal{H}_{1}, \mathcal{H}_{2}$ are Hopf $*$-algebras and $\gamma$ is their $*$-epimorphism, then $H_{2} \backslash H_{1}, H_{1} / H_{2}, H_{2} \backslash H_{1} / H_{2}$ are unital $*$-algebras, $\Delta \circ *=(* \otimes *) \circ \Delta$ and $\Delta$ maps the cone of positive elements of $H_{2} \backslash H_{1} / H_{2}$ to the cone of positive elements of its tensor product.

Now recall that if $\mathcal{H}_{1}$ is a Hopf *-algebra associated with a compact quantum group, then [29] $H_{1}$ can be represented as

$$
H=\sum_{\alpha} \sum_{i, j=1}^{d_{\alpha}} \mathrm{C} u_{i, j}^{\alpha},
$$


where $u_{i, j}^{\alpha}$ are matrix elements of $d_{\alpha}$-dimensional unitary corepresentation of $H_{1}\left(d_{\alpha}<\infty\right.$ for all $\alpha$ running over some discrete set $\left.\hat{Q}\right)$ and there exists an invariant integral $\nu$ on $H$, which is a state and such that $\alpha$-sum in (12) defines an orthogonal decomposition in the sense of the inner product given by $\langle f, g\rangle:=\nu\left(f \cdot g^{*}\right)$ after a suitable choice of an orthonormal basis for each representation space. In this case, the comodules $H_{2} \backslash H_{1}, H_{1} / H_{2}$ as well as $H_{2} \backslash H_{1} / H_{2}$ may be given by

$$
\begin{gathered}
H_{2} \backslash H_{1}=\sum_{\alpha} \sum_{i=1}^{d_{\alpha}^{\prime}} \sum_{j=1}^{d_{\alpha}} \mathrm{C} u_{i, j}^{\alpha}, H_{1} / H_{2}=\sum_{\alpha} \sum_{i=1}^{d_{\alpha}} \sum_{j=1}^{d_{\alpha}^{\prime}} \mathrm{C} u_{i, j}^{\alpha}, \\
H_{2} \backslash H_{1} / H_{2}=\sum_{\alpha} \sum_{i, j=1}^{d_{\alpha}^{\prime}} \mathrm{C} u_{i, j}^{\alpha}
\end{gathered}
$$

where $d_{\alpha}^{\prime} \leq d_{\alpha}$ for all $\alpha$. One can see that

$$
\Delta\left(u_{i, j}^{\alpha}\right)=\sum_{k=1}^{d_{\alpha}^{\prime}} u_{i, k}^{\alpha} \otimes u_{k, j}^{\alpha} .
$$

\section{Quantum Stiefel manifold $U_{q}(n) / U_{q}(n-m)$.}

3.1.

For studying the structure of the quantum space $U_{q}(n) / U_{q}(n-m)$, decompose $U_{q}(n)$ into a direct sum of irreducible $U_{q}(n-m)$-comodules.

Definition 4. A dominant integral weight $\mu \in L_{i}$ is said to be subordinated to a dominant integral weight $\nu \in L_{j}(1 \leq i<j \leq n)$ if

$$
\nu_{k+j-i} \leq \mu_{k} \leq \nu_{k} \quad(\forall 1 \leq k \leq i) .
$$

In this case we write $\mu \prec \nu$.

Lemma 1. Let $\lambda$ be a dominant integral weight in $L_{n}, V^{R}(\lambda)$ be an irreducible right $U_{q}(n)$-comodule of the weight $\lambda$. Considering $V^{R}(\lambda)$ as a right $U_{q}(n-m)$-comodule $(1 \leq m \leq n)$ with a coaction $R_{U_{q}(n-m)}:=$ $\left(\mathrm{id} \otimes \gamma_{m}\right) R_{U_{q}(n)}$, we have a decomposition:

$$
V^{R}(\lambda) \sim \oplus_{\mu^{n-m}} K_{\mu^{n-m}}^{\lambda} V^{R}\left(\lambda, \mu^{n-m}\right),
$$

where $\mu^{n-m}$ runs over the set of all dominant integral weights in $L_{n-m}$ subordinated to $\lambda, V^{R}\left(\lambda, \mu^{n-m}\right)$ is an irreducible $U_{q}(n-m)$-comodule of the weight $\mu^{n-m}, K_{\mu^{n-m}}^{\lambda} \geq 1$ is its multiplicity, $\sim$ means an isomorphism.

Proof. For $m=1$ the result has been obtained in [14]:

$$
V^{R}(\lambda)=\oplus_{\mu^{n-1}} V^{R}\left(\lambda, \mu^{n-1}\right) .
$$


Every $U_{q}(n-1)$-subcomodule $V^{R}\left(\lambda, \mu^{n-1}\right)$ of $V^{R}(\lambda)$ is isomorphic to an irreducible right $U_{q}(n-1)$-comodule $V^{R}\left(\mu^{n-1}\right)$ with weight $\mu^{n-1}$. By this isomorphism and a decomposition of $V^{R}\left(\mu^{n-1}\right)$ similar to (16), on the second step we have

$$
V^{R}(\lambda)=\oplus_{\mu^{n-1}} \oplus_{\mu^{n-2}} V^{R}\left(\lambda, \mu^{n-1}, \mu^{n-2}\right) \sim \oplus_{\{\mu\}^{n-2}} V^{R}\left(\lambda,\{\mu\}^{n-2}\right),
$$

where $\{\mu\}^{n-2}$ is the collection of the weights $\left\{\mu^{n-1}, \mu^{n-2}\right\}$ with $\mu^{n-2} \prec$ $\mu^{n-1}$. $V^{R}\left(\lambda,\{\mu\}^{n-2}\right)$ is a $U_{q}(n-2)$-subcomodule of the right $U_{q}(n-2)$ comodule $V^{R}(\lambda)$, and $V^{R}\left(\lambda,\{\mu\}^{n-2}\right)$ is isomorphic to an irreducible right $U_{q}(n-2)$-comodule of the weight $\mu^{n-2}$. On the $m$-th step of this process one gets a decomposition:

$$
V^{R}(\lambda)=\oplus_{\{\mu\}^{n-m}} V^{R}\left(\lambda,\{\mu\}^{n-m}\right),
$$

where $\{\mu\}^{n-m}$ runs over the set of all collections of the dominant integral weights $\{\mu\}^{n-m}=\left\{\mu^{n-1}, \cdots, \mu^{n-m}\right\}, \quad \mu^{i} \in L_{i}$, with $\mu^{i-1} \prec \mu^{i}$.

Here $V^{R}\left(\lambda,\{\mu\}^{n-m}\right)$ is a $U_{q}(n-m)$-subcomodule of the right $U_{q}(n-m)$ comodule $V^{R}(\lambda)$ and it is isomorphic to an irreducible $U_{q}(n-m)$-comodule of the weight $\mu^{n-m}$. There are several collections $\{\mu\}^{n-m}$ "leading" from $\lambda$ to $\mu^{n-m}$; the number of these collections defines the corresponding multiplicity $K_{\mu^{n-m}}^{\lambda}$ (the formula for computation of this number is quite complicated).

\section{2.}

In a usual way, one can give a definition of the highest vector of the weight $\mu^{n-i}$ in the irreducible $U_{q}(n-i)$-comodule $V^{R}\left(\lambda, \mu^{n-i}\right)$. It can be constructed as follows. Remind that the basis in $V^{R}(\lambda)$ is formed by all possible standard monomials $\xi_{T}$ indexed by the semi-standard tableaus $T$ in $\operatorname{SSTab}_{n}(\lambda)$.

(1) Let a tableau $T$ be such that $\xi_{T}$ is the highest vector of an irreducible $U_{q}(n-i)$-comodule. This is possible if and only if $e_{k} \cdot \xi_{T}=0 \forall k \in\{1, \ldots, n-$ $i-1\}$. Since $e_{k} \cdot \xi_{j_{1} \cdots j_{s}}=\xi_{j_{1} \cdots j_{p}-1 \cdots j_{s}}$, if some $j_{p}=k+1$, and $e_{k} \cdot \xi_{j_{1} \cdots j_{s}}=0$, if $j_{1}, \cdots, j_{s} \neq k+1\left(j_{1} \leq \cdots \leq j_{s} \leq n\right)$, then one gets that

$$
e_{k} \cdot \xi_{J_{s}}=0, \forall k \in\{1, \cdots, n-i-1\},
$$

if $J_{s}=\left(1, \cdots, p_{s}, j_{p_{s}+1}, \cdots, j_{s}\right)$, where $1 \leq p_{s} \leq n-i, j_{p_{s}+1}, \cdots j_{s} \geq n-i$. So the above structure of the lines of the tableau $T$ is necessary. After that it remains to check that for such a tableau we also have:

$$
q^{\varepsilon_{k}} \cdot \xi_{T}=q^{\mu_{k}^{n-i}} \xi_{T}, \varepsilon_{k} \in L_{n-i}, \forall k=\overline{1, n-i} .
$$

(2) If a tableau $T$ has the above property, one can get a collection of tableaus $T^{(n-i)}(1 \leq i \leq n$ ) (each of them is obtained by removing from $T$ its elements $T_{r s}$ exceeding $\left.n-i\right)$. Every $r$-column of the tableau $T^{(n-i)}$ has, obviously, the height $\mu_{r}^{n-i}=\operatorname{card}\left\{1 \leq s \leq \lambda_{r} \mid T_{r s} \leq n-i\right\}$, and it is clear that $\mu^{n-i}=0$ if $r \geq n-i$. So, $\mu^{n-i}=\sum_{r=1}^{n-i} \mu_{r}^{n-i} \varepsilon_{r} \in L_{n-i}$, and the 
collection of the weights $\{\mu\}^{n-i}$ satisfies the condition of subordination. Let us show that the monomial $\xi_{T^{(n-i)}}$ is the highest vector of the weight $\mu^{n-i}$ in the irreducible $U_{q}(n-i)$-comodule.

In fact, according to the construction of $T^{(n-i)}$, one has $e_{k} \cdot \xi_{T^{(n-i)}}=0$ $\forall k \in\{1, \cdots, n-i-1\}$. Now it is sufficient to show that $q^{\varepsilon_{k}} \cdot \xi_{T^{(n-i)}}=$ $q^{\mu_{k}^{n-i}} \xi_{T^{(n-i)}} \forall k \in\{1, \cdots, n-i\}$. Taking into consideration the formulae $q^{\varepsilon_{k}}$. $t_{i j}=q^{\delta_{k j}} t_{i j}, q^{\varepsilon_{k}} \cdot \xi_{J}=q^{\left\langle\varepsilon_{k}, \Lambda_{J}\right\rangle} \xi_{J}$ and $q^{\varepsilon_{k}} \cdot \xi_{T^{(n-i)}}=q^{\left\langle\varepsilon_{k}, \Lambda_{J_{1}^{n-i}}+\cdots+\Lambda_{J_{s}^{n-i}}\right\rangle} \xi_{T^{(n-i)}}$ $=q^{\mu_{k}^{n-i}} \xi_{T^{(n-i)}}$. Considering a line $J$ and the corresponding line $J^{(n-i)}$, we have $q^{\varepsilon_{k}} \cdot \xi_{J}=q^{\left\langle\varepsilon_{k}, \Lambda_{J}\right\rangle} \xi_{J}=q^{\left\langle\varepsilon_{k}, \Lambda_{J}(n-i)\right.} \xi_{J} \forall k \in\{1, \cdots, n-i\}$. This means that $q^{\varepsilon_{k}} \cdot \xi_{T}=q^{\left\langle\varepsilon_{k}, \Lambda_{J_{1}^{n-i}+\cdots+\Lambda} J_{s}^{n-i}\right\rangle} \xi_{T}=q^{\mu_{k}^{n-i}} \xi_{T}$.

So one gets that $\xi_{T}$ (corresponding to the semi-standard tableau $T$ with the above property) is the highest vector of the weight $\mu_{r}^{(n-i)}$ in the irreducible $U_{q}(n-i)$-comodule $V^{R}(\lambda)$. The epimophism $\gamma_{i}: U_{q}(n) \rightarrow U_{q}(n-i)$ maps $\xi_{T}$ to $\xi_{T^{(n-i)}}$ and in this way generates an isomorphism of irreducible $U_{q}(n-i)$-comodules.

Remark 3. Lemma 1 and the mentioned construction of the highest vector can be applied also to left comodules.

\section{3.}

In what follows we consider the homogeneous space $U_{q}(n) / U_{q}(n-m)$, i.e., the subcomodule $V^{R}(\lambda)$ of comodule $U_{q}(n)$ of the weight $\mu^{n-m}=0$. From inequalities $0=\mu_{1}^{n-m} \geq \mu_{2}^{n-m-1} \geq \cdots \geq \mu_{n-m}^{1} \geq \lambda_{n-m+1} \geq \cdots \geq \lambda_{n}$ we obtain $\lambda_{n} \leq 0$. The condition $\mu^{n-m}=0$ means that the tableaus $T \in$ $\operatorname{SSTab}_{n}(\lambda)$ do not contain the numbers $1, \cdots, n-m$. Using Proposition 3 , we can proceed to the case $\lambda_{n} \geq 0$. The new weights can be written as: $\tilde{\lambda}=\lambda-\lambda_{m}\left(\varepsilon_{1}+\cdots+\varepsilon_{n}\right), \tilde{\mu}^{n-m}=\mu^{n-m}-\lambda_{n}=-\lambda_{n}$. That is why the new tableaus $T \in S S T a b_{n}\left(\lambda-\lambda_{n}\left(\varepsilon_{1}+\cdots+\varepsilon_{n}\right)\right)$ corresponding to the highest weights of this comodule will already contain the numbers $1, \cdots, n-m$. Moreover, each of them should contain a rectangle block beginning from the first line and the first column, every its line containing a completely ordered set $1, \cdots, n-m$, the number of its columns being equal to $-\lambda_{n}$.

Consider now the generators $v_{T}=\operatorname{det}_{q}^{\lambda_{n}} \xi_{T}$ of the above $U_{q}(n-m)$ comodule. Since the number of lines of $T$ containing the numbers $1, \cdots, n-$ $m$ equals to $-\lambda_{n}$, then these generators can be written in the form: $v_{T}=$ $\operatorname{det}_{q}^{-1} \xi_{T_{1}} \cdots \operatorname{det}_{q}^{-1} \xi_{T_{-\lambda_{n}}} \xi_{T_{-\lambda_{n}+1}} \cdots \xi_{T_{\lambda_{1}-\lambda_{n}}}$, where $T_{i}$ are lines of $T$. By the definition of $*$ we have: $\operatorname{det}_{q}^{-1} \xi_{T_{i}}=\operatorname{sgn}_{q}\left(T_{i}, T_{i}^{c}\right)\left(\xi_{T_{i}^{c}}^{I_{i}}\right)^{*}$ and then $v_{T}=$ $\left(\xi_{T_{1}^{c}}^{I_{1}}\right)^{*} \cdots\left(\xi_{T_{-\lambda_{n}}^{c}}^{I_{-\lambda_{n}}}\right)^{*} \cdot \xi_{T_{-\lambda_{n}+1}} \cdots \xi_{T_{\lambda_{1}-\lambda_{n}}}$. Here $T_{i}^{c}$ is the complement of $T_{i}$ to the set $(1, \cdots, n), I_{i}$ is the line $(p, \cdots, n), p$ is the length of the $T_{i}$, and $\operatorname{sgn}_{q}(I, J)=(-q)^{l(I, J)}, l(I, J)=\#\{(i, j): i \in I, j \in J, i>j\}$. 
The lines $T_{-\lambda_{n}+1}, \cdots, T_{\lambda_{1}-\lambda_{n}}$ do not contain the numbers $1, \cdots, n-m$ by the construction.

The comodule generated by the vectors $v_{T}$ is the $U_{q}(n-m)$-subcomodule of the weight $\mu^{n-m}=0$ in $U_{q}(n-m)$-comodule $V^{R}(\lambda)$. We denote it by $V^{R}(\lambda)_{n-m}^{0}$. There is an isomorphism $W(\lambda) / U_{q}(n-m) \sim V^{L}(\lambda) \otimes V^{R}(\lambda)_{n-m}^{0}$ and $U_{q}(n) / U_{q}(n-m) \sim \oplus_{\lambda} V^{L}(\lambda) \otimes V^{R}(\lambda)_{n-m}^{0}$, where $\lambda$ runs over the set of all weights such that $\mu^{n-m}=0 \prec \lambda$. This isomorphism imposes a restriction on lower indices of the minors generating $W(\lambda) / U_{q}(n-m)$.

Thus, the algebraic generators of $U_{q}(n) / U_{q}(n-m)$ are the minors $\xi_{j_{1}, \cdots j_{r}}^{i_{1}, \cdots i_{r}}$ and $\left(\xi_{j_{1}, \cdots j_{r}}^{i_{1}, \cdots i_{r}}\right)^{*}\left(1 \leq i_{1}<\cdots<i_{r} \leq n ; n-m+1 \leq j_{1}<\cdots<j_{r} \leq n ; 1 \leq r \leq\right.$ $m)$. These minors are polynomials in $t_{i j} ; t_{i j}^{*}(1 \leq i \leq n, n-m+1 \leq j \leq n)$.

The result of our considerations can be summarized as follows:

Theorem 1. 1) The quantum homogeneous space $S_{q}^{n, m}=U_{q}(n) / U_{q}(n-m)$ is the algebra generated by $t_{i j}, t_{i j}^{*}(1 \leq i \leq n, n-m+1 \leq j \leq n)$ satisfying the following relations:

$$
t_{i k} t_{j k}=q t_{j k} t_{i k}, \quad t_{k i} t_{k j}=q t_{k j} t_{k i} \quad(i<j) \quad t_{i l} t_{j k}=t_{j k} t_{i l} \quad(i<j, k<l)
$$

$$
t_{i k} t_{j l}-q t_{i l} t_{j k}=t_{j l} t_{i k}-q^{-1} t_{j k} t_{i l}(i<j, k<l)
$$

$t_{i k}^{*} t_{j k}^{*}=q^{-1} t_{j k}^{*} t_{i k}^{*}, \quad t_{k i}^{*} t_{k j}^{*}=q^{-1} t_{k j}^{*} t_{k i}^{*} \quad(i<j) \quad t_{i l}^{*} t_{j k}^{*}=t_{j k}^{*} t_{i l}^{*} \quad(i<j, k<l)$

$$
\begin{gathered}
t_{i k}^{*} t_{j l}^{*}-q^{-1} t_{i l}^{*} t_{j k}^{*}=t_{j l}^{*} t_{i k}^{*}-q t_{j k}^{*} t_{i l}^{*} \quad(i<j, k<l) \\
q t_{l p} t_{l p}^{*}+\left(q-q^{-1}\right) \sum_{m>p} t_{l m} t_{l m}^{*}=q t_{l p}^{*} t_{l p}+\left(q-q^{-1}\right) \sum_{r<l} t_{r p}^{*} t_{r p} \\
t_{i j} t_{i s}^{*}=q t_{i s}^{*} t_{i j}+\left(q-q^{-1}\right) \sum_{p<i} t_{p s}^{*} t_{p j} \quad(s \neq j) \\
q t_{l p} t_{j p}^{*}+\left(q-q^{-1}\right) \sum_{m>p} t_{l m} t_{j m}^{*}=t_{j p}^{*} t_{l p}(l \neq j) \\
t_{k j} t_{p s}^{*}=t_{p s}^{*} t_{k j}(k \neq p, j \neq s) \\
\sum_{i=1}^{n} t_{i l} t_{i j}^{*}=\delta_{l j} .
\end{gathered}
$$

2) The relations (17), (18), (19), (20) form the full system of relations between the generators $t_{i k}, t_{i k}^{*}(1 \leq i \leq n, n-m+1 \leq k \leq n)$ of the algebra $S_{q}^{n, m}$. 
Proof. 1) It was explained already that the algebra $S_{q}^{n, m}$ is generated by $t_{i k}, t_{i k}^{*}(1 \leq i \leq n, n-m+1 \leq k \leq n)$. We shall prove the formulae (17)-(20) using the matrices $\mathbf{T}=\left(t_{i j}\right)_{i, j=1}^{n}$ and $\mathbf{T}^{*}=\left(t_{j i}^{*}\right)_{i, j=1}^{n}$.

The formula (20) follows from the equation $\mathbf{T} \mathbf{T}^{*}=\mathbf{T}^{*} \mathbf{T}=I$ (see 2.6). The formula (17) follows from the Equation (6): $(\mathbf{T} \otimes \mathbf{T}) \mathbf{R}=\mathbf{R}(\mathbf{T} \otimes \mathbf{T})$, where $\mathbf{T} \otimes \mathbf{T}=(\mathbf{T} \otimes I)(I \otimes \mathbf{T})$. Multiplying both sides by $\left(\mathbf{T}^{*} \otimes I\right)$ on the left, one gets $(I \otimes \mathbf{T}) \mathbf{R}=\left(\mathbf{T}^{*} \otimes I\right) \mathbf{R}(\mathbf{T} \otimes I)(I \otimes \mathbf{T}) ;$ multiplying both sides by $I \otimes \mathbf{T}^{*}$ on the right, one gets $(I \otimes \mathbf{T}) \mathbf{R}\left(I \otimes \mathbf{T}^{*}\right)=\left(\mathbf{T}^{*} \otimes I\right) \mathbf{R}(\mathbf{T} \otimes I)$.

The coordinate form of this formula gives us (19). And, finally, multiplying the last line by the $I \otimes \mathbf{T}^{*}$ on the right and by the $\mathbf{T}^{*} \otimes I$ on the left, one gets $\mathbf{R}\left(I \otimes \mathbf{T}^{*}\right)\left(\mathbf{T}^{*} \otimes I\right)=\left(I \otimes \mathbf{T}^{*}\right)\left(\mathbf{T}^{*} \otimes I\right) \mathbf{R}$ or $\mathbf{R}\left(\mathbf{T}^{*} \otimes \mathbf{T}^{*}\right)=\left(\mathbf{T}^{*} \otimes \mathbf{T}^{*}\right) \mathbf{R}$. From this formula we obtain (18).

2) It is known that monomials in $t_{i k}, t_{i k}^{*}(1 \leq i \leq n, 1 \leq k \leq n)$ having the lexicographic order form a basis in $U_{q}(n)[\mathbf{1 4}]$, [18]. Similarly one can show, using the relations $(17),(18),(19),(20)$, that monomials in $t_{i k}, t_{i k}^{*}(1 \leq$ $i \leq n, n-m+1 \leq k \leq n)$ having the lexicographic order form a basis in $S_{q}^{n, m}$. This allows us to identify the algebra generated by the above letters and relations with certain subalgebra of $U_{q}(n)$. After that it is clear that there is no nontrivial polynomial in $t_{i k}, t_{i k}^{*}(1 \leq i \leq n, n-m+1 \leq k \leq n)$ equal to 0.

Remark 4. The similar facts are valid for $U_{q}(n-m) \backslash U_{q}(n)$. In the classical case $q=1$ the homogeneous space above is called a Stiefel manifold. That is why in what follows we call $S_{q}^{n, m}=U_{q}(n-m) / U_{q}(n)$ a quantum Stiefel manifold.

\section{Irreducible representations of $S_{q}^{n, m}$.}

4.1.

Recall some basic facts referring to representations of the algebra of polynomials on the quantum group $S U_{q}(n)[\mathbf{2 0}],[\mathbf{1 9}]$. The algebra of polynomials on $S U_{q}(2)$ has the following irreducible representations: one-dimensional $\chi_{\phi}\left(s_{11}\right)=e^{i \phi}, \chi_{\phi}\left(s_{22}\right)=e^{-i \phi}, \chi_{\phi}\left(s_{12}\right)=\chi_{\phi}\left(s_{21}\right)=0(\phi \in[0,2 \pi))$ and an infinite-dimensional $\rho_{0}$ in $l_{2}\left(\mathbf{Z}_{+}\right): \rho_{0}\left(s_{11}\right) e_{0}=0, \rho_{0}\left(s_{11}\right) e_{k}=\left(1-q^{2 k}\right)^{1 / 2} e_{k-1}$ $k \geq 1 ; \rho_{0}\left(s_{21}\right) e_{k}=-q^{k} e_{k} ; \rho_{0}\left(s_{22}\right)=\rho_{0}\left(s_{11}\right)^{*} ; \rho_{0}\left(s_{12}\right)=-q \rho_{0}\left(s_{21}\right)$ (from here on we consider only $q \in(0,1))$.

The representations of $S U_{q}(n)$ can be constructed using the above representations of $S U_{q}(2)$. Let $\psi_{i}: U_{q}(s l(2)) \rightarrow U_{q}(s l(n))$ be the inclusion of the quantum enveloping algebras such that $\psi_{i}(e)=e_{i} ; \psi_{i}(f)=f_{i} ; \psi_{i}\left(q^{\varepsilon}\right)=$ $q^{\varepsilon_{i}} \quad 1 \leq i \leq n-1$. Then $\psi_{i}^{*}: S U_{q}(n) \rightarrow S U_{q}(2)$ and $\pi_{i}=\rho_{0} \psi_{i}^{*}$ are the irreducible $*$-representations of $S U_{q}(n)$. By this construction, $\pi_{i}\left(t_{i i}\right)$ and $\pi_{i}\left(t_{i+1, i+1}\right)$ contain the shift operators, $\pi_{i}\left(t_{i, i+1}\right)$ and $\pi_{i}\left(t_{i+1, i}\right)$ are diagonal and $\pi_{i}\left(t_{k, l}\right)=\delta_{k l} 1$ for all other generators. 
Let $S_{n} \ni \omega=\tau_{i_{1}} \cdots \tau_{i_{k}}$ be a decomposition of some element $\omega$ of the permutation group, which has the least possible number of transpositions $\tau_{j}=(j, j+1)$. The representation $\pi_{\omega}=\pi_{i_{1}} \otimes \cdots \otimes \pi_{i_{k}}$ corresponds to the element $\omega$ (recall that if $\mathcal{H}$ is a Hopf algebra, $\rho_{1}$ is a representation of its algebra $H, A$ is a right comodule algebra over $\mathcal{H}$ with respect to the coaction $R_{A}: H \rightarrow H \otimes A, \rho_{2}$ is a representation of the algebra $A$, then one can construct a new representation of the algebra $\left.H: \rho_{1} \otimes \rho_{2}:=\left(\rho_{1} \otimes \rho_{2}\right) \circ R_{A}\right)$.

Proposition 8. The *-representations $\pi_{\omega}$ are irreducible and any irreducible *-representation of $S U_{q}(n)$ is equivalent to some $\pi_{\omega}$ up to a onedimensional tensor factor.

Remark 5. A similar statement is also valid for $U_{q}(n)$. The only difference is that the set of one-dimensional representations of the group $S U_{q}(n)$ is $(n-1)$-parametric and of the group $U_{q}(n)$ is $n$-parametric.

\section{2.}

One can construct an irreducible $*$-representation of the quantum Stiefel manifold $S_{q}^{n, m}=U_{q}(n) / U_{q}(n-m)=S U_{q}(n) / S U_{q}(n-m)$ using these facts. Let us consider cosets $S_{n} / S_{n-m}$ of the permutation group $S_{n}$ with respect to the subgroup $S_{n-m}$. Chose from every coset an element having the least possible length $\omega=\tau_{j_{1}} \cdots \tau_{j_{l}}$.

Theorem 2. Representations $\pi_{\omega}$ of $S_{q}^{n, m}$ corresponding to elements $\omega$ of the least possible length, among the representatives of the class from $S_{n} / S_{n-m}$, are irreducible.

Proof. Let us consider the structure of elements of $S_{n}$ (resp., $S_{n} / S_{n-m}$ ) and the structure of the corresponding representations of $U_{q}(n)$ (resp., $S-q^{n, m}$ ). An arbitrary element of $S_{n}$ can be written as follows:

$$
\begin{gathered}
\omega=\tau_{i_{1}} \cdots \tau_{n-1} \tau_{i_{2}} \cdots \tau_{n-2} \cdots \tau_{i_{k}} \cdots \tau_{n-k}, \\
\left(1 \leq k \leq n-1, i_{1} \leq \cdots \leq n-1, \cdots, i_{k} \leq \cdots \leq n-k\right),
\end{gathered}
$$

(in particular, the greatest element $\omega_{0} \in S_{n}$ has the form:

$$
\left.\omega_{0}=\tau_{1} \tau_{2} \cdots \tau_{n-1} \tau_{1} \cdots \tau_{n-2} \cdots \tau_{1} \tau_{2} \tau_{1}\right) .
$$

The corresponding series of representations of $U_{q}(n)$

$$
\pi_{\omega, \bar{\phi}}=\pi_{i_{1}} \otimes \cdots \otimes \pi_{n-1} \otimes \pi_{i_{2}} \otimes \cdots \otimes \pi_{n-2} \otimes \cdots \otimes \pi_{i_{k}} \otimes \cdots \otimes \pi_{n-k} \otimes \kappa_{\bar{\phi}},
$$

is parametrized by one-dimensional representations $\kappa_{\bar{\phi}}$. Here one-dimensional representations $\kappa_{\bar{\phi}}$ correspond to the greatest torus in $U_{q}(n)$.

Respectively, an arbitrary representative of a class from $S_{n} / S_{n-m}$ having the least possible length can be written as follows:

$$
\omega=\tau_{i_{1}} \cdots \tau_{n-1} \tau_{i_{2}} \cdots \tau_{n-2} \cdots \tau_{i_{k}} \cdots \tau_{n-k} \quad(1 \leq k \leq m),
$$


(in particular, the greatest element from $S_{n} / S_{n-m}$, the representative of the class $\omega_{0} S_{n-m}$ having the least possible length, is

$$
\left.\omega_{m}=\tau_{1} \tau_{2} \cdots \tau_{n-1} \tau_{1} \cdots \tau_{n-2} \cdots \tau_{1} \cdots \tau_{n-m}\right) .
$$

The corresponding series of representations of $S_{q}^{n, m}$

$$
\pi_{\omega, \bar{\phi}}=\pi_{i_{1}} \otimes \cdots \otimes \pi_{n-1} \otimes \pi_{i_{2}} \otimes \cdots \otimes \pi_{n-2} \otimes \cdots \otimes \pi_{i_{k}} \otimes \cdots \otimes \pi_{n-k} \otimes \kappa_{\bar{\phi}},
$$

is parametrized by one-dimensional representations corresponding to the greatest torus in $S_{q}^{n, m}$.

Now let us show that every representation $\pi_{\omega}$ is irreducible. The irreducibility of this representation follows from the fact that for any tensor component of $\pi_{\omega}(\cdot)$ one can find such a generator $t$ of $S_{q}^{n, m}$ for which this tensor component of $\pi_{\omega}(t)$ containes the shift operator. More precisely, one can see that for any tensor component of $\pi_{\omega}(\cdot)$ with a number from the group $\left\{i_{l}, \ldots, n-l\right\}(l=1, \ldots, k)$ it is possible to find such a generator $t$ from the group $\left\{t_{1, n-l+1}, \ldots, t_{n, n-l+1}\right\}$ for which this tensor component of $\pi_{\omega}(t)$ containes the shift operator. Let us show the typical consideration supporting this statement. Let us consider, for example, the greatest representation $\pi_{\omega_{m}}$. Then

$$
\begin{aligned}
\pi_{\omega_{m}}\left(t_{i n}\right)= & \pi_{1}\left(t_{i i}\right) \otimes \cdots \otimes \pi_{i-1}\left(t_{i i}\right) \otimes \pi_{i}\left(t_{i, i+1}\right) \otimes \cdots \otimes \pi_{n-1}\left(t_{n-1, n}\right) \otimes \pi_{1}\left(t_{n n}\right) \otimes \cdots \\
& \otimes \pi_{n-2}\left(t_{n n}\right) \otimes \cdots \otimes \pi_{1}\left(t_{n n}\right) \otimes \pi_{n-m}\left(t_{n n}\right) \quad(1 \leq i \leq n) .
\end{aligned}
$$

The operator $\pi_{i-1}\left(t_{i i}\right)$ containes the shift operator by the construction. The operators $\pi_{j}\left(t_{j, j+1}\right)(i \leq j \leq n-1)$ are diagonal and all the others are unit operators. Hence, the above statement is true for the tensor components of $\pi_{\omega_{m}}(\cdot)$ with numbers from the group $\{1, \ldots, n-1\}$. Similarly one can consider all other cases. Thus, the proof is completed.

Theorem 3. Each irreducible *-representation $\rho$ of $S_{q}^{n, m}$ is equivalent (up to a one-dimensional tensor factor) to one of the representations $\pi_{\omega}$, where $\omega$ has the least possible length among the representatives of the class from $S_{n} / S_{n-m}$.

Proof. Continue $\rho$ up to the representation of the quantum group $S U_{q}(n)$ in the following way: $\rho\left(t_{i j}\right)=\rho\left(t_{i j}^{*}\right)=\delta_{i j} 1(1 \leq j \leq n-m, 1 \leq i \leq n)$. It is irreducible because it is irreducible on the subalgebra. Then, according to Proposition 8 , it is equivalent to the representation $\pi_{i_{1}} \otimes \cdots \otimes \pi_{i_{k}} \otimes \kappa_{\phi}\left(\kappa_{\phi}\right.$ is one-dimensional representation).

Let us consider the permutation $\omega=\tau_{i_{1}} \cdots \tau_{i_{k}}$ corresponding to this representation. The representation $\rho$ and, consequently, $\pi_{\omega}$ are irreducible on $S_{q}^{n, m}$. Then let us show that $\pi_{\omega}$ is irreducible only if $\omega$ has the least possible length. Consider a transposition $\tau_{s} \in S_{n-m}$, a representation $\pi_{\omega} \otimes \pi_{s}=\pi_{i_{1}} \otimes \cdots \otimes \pi_{i_{k}} \otimes \pi_{s}$ and its action on the generators $t_{i k}(1 \leq$ $i \leq n, n-m+1 \leq k \leq n)$ of $S_{q}^{n, m}: \pi_{\omega} \otimes \pi_{s}\left(t_{i k}\right)=\sum_{r=1}^{n} \pi_{\omega}\left(t_{i r}\right) \otimes \pi_{s}\left(t_{r k}\right)$. But $\pi_{s}\left(t_{r k}\right)=\delta_{r k} 1(1 \leq r \leq n, n-m+1 \leq k \leq n)$ by the construction of 
the representations $\pi_{s}(s \leq n-m-1)$. So $\pi_{\omega} \otimes \pi_{s}\left(t_{i k}\right)=\pi_{\omega}\left(t_{i k}\right) \otimes 1$ and this representation is not irreducible. Thus we have proved that the length of $\omega$ should be the least possible.

\section{3.}

Let us consider now the set of representations $\pi_{\omega, \bar{\phi}}=\pi_{\omega} \otimes \kappa_{\bar{\phi}}$, where $\kappa_{\bar{\phi}}$ are one-dimensional representations of $S_{q}^{n, m}$ and $\omega \in S_{n} / S_{n-m}$.

Theorem 4. For the representations of $S_{q}^{n, m}$ we have:

$$
\cap_{\omega \in S_{n} / S_{n-m}, \bar{\phi}} \operatorname{Ker} \pi_{\omega, \bar{\phi}}=0 .
$$

Proof. Let $\cap_{\omega \in S_{n} / S_{n-m}, \bar{\phi}} \operatorname{Ker} \pi_{\omega, \bar{\phi}}=L$. It was shown in the proof of Theorem 3 that $\left(\pi_{\omega, \bar{\phi}} \otimes \pi_{\sigma}\right)(x)=\left(\pi_{\omega, \bar{\phi}} \otimes 1\right)(x) \forall \sigma \in S_{n-m} \forall x \in S_{q}^{n, m}$. So for $x \in$ $L$ we have $\left(\pi_{\omega, \bar{\phi}} \otimes \pi_{\sigma}\right)(x)=0$. Taking all $\sigma \in S_{n-m}$, one can obtain all the representations of $U_{q}(n)$. Thus, $L=\cap_{\gamma \in S_{n},{ }_{\phi}} \operatorname{Ker} \pi_{\gamma, \bar{\phi}}=0$, because $\pi_{\gamma, \bar{\phi}}$ $\left(\gamma \in S_{n}\right)$ give all the representations of $U_{q}(n)$, and it is known [28] that the intersection of their kernels is 0 .

Now one can construct a $C^{*}$-algebra $\mathbf{C}\left(S_{q}^{n, m}\right)$ of the quantum Stiefel manifold $S_{q}^{n, m}$, considering the completion of $S_{q}^{n, m}$ with respect to the $C^{*}$ norm

$$
\|\cdot\|=\sup _{\omega, \bar{\phi}}\left\|\pi_{\omega, \bar{\phi}}(\cdot)\right\| .
$$

Remark 6. The $C^{*}$-algebra $\mathbf{C}\left(U_{q}(n)\right)$ of the quantum group $U_{q}(n)$ was introduced in a number of papers, for example, in [18], [29]. The $C^{*}$-algebra $\mathbf{C}\left(S_{q}^{n, 1}\right)$ was introduced in [17], [18], [28].

\section{Invariant integral.}

\section{1 .}

The existence and the uniqueness of the invariant integral $\nu_{n}$ on $U_{q}(n)$ are known $(2.5,2.6)$. Our aim is to obtain a formula for its calculation as well as a similar formula for an invariant integral $\nu_{n, n-m}$ on $S_{q}^{n, m}$ (i.e., a linear functional on $S_{q}^{n, m}$ such that (id $\left.\otimes \nu_{n, n-m}\right) \circ \Delta(f)=\nu_{n, n-m}(f) 1$ for every $\left.f \in S_{q}^{n, m}\right)$. We shall do it by induction in $n$. For this we use the formula for the invariant integral $\nu_{n, n-1}$ on $S_{q}^{n, 1}=S U_{q}(n) / S U_{q}(n-1)=U_{q}(n) / U_{q}(n-1)$ which has been obtained in [28]:

$$
\nu_{n, n-1}(f)=(2 \pi)^{-1} \int_{0}^{2 \pi} \operatorname{tr}\left(\pi_{\omega_{1}, \phi}(f) Q\right) d \phi,
$$

where $\omega_{1}$ is the greatest element in $S_{n} / S_{n-1}, Q: l_{2}\left(\mathbf{Z}_{+}\right)^{\otimes(n-1)} \rightarrow$ $l_{2}\left(\mathbf{Z}_{+}\right)^{\otimes(n-1)}$ is a linear operator

$$
Q\left(e_{m_{1}} \otimes \cdots \otimes e_{m_{n-1}}\right)=Q\left(m_{1}, \ldots, m_{n-1}\right) e_{m_{1}} \otimes \cdots \otimes e_{m_{n-1}},
$$


where $Q\left(m_{1}, \ldots, m_{n-1}\right)=\prod_{j=1}^{n-1}\left(1-q^{2(n-j)}\right)^{-1} q^{2 \sum_{j=1}^{n-1} m_{j}(n-j)}$. One can see that the operator $Q$ is equal to the operator

$$
\begin{gathered}
\pi_{1}\left(t_{12}^{*} t_{12}\right)\left(1-q^{2}\right)^{-1} \otimes \pi_{2}\left(\left(t_{23}^{*} t_{23}\right)^{2}\right)\left(1-q^{4}\right)^{-1} \otimes \cdots \\
\cdots \otimes \pi_{n-1}\left(\left(t_{n-1, n}^{*} t_{n-1, n}\right)^{n-1}\right)\left(1-q^{2(n-1)}\right)^{-1}
\end{gathered}
$$

and its trace equals to 1 .

Lemma 2. The formula for the invariant integral on $U_{q}(n)$ is:

$$
\nu_{n}(f)=(2 \pi)^{-n} \int_{T_{0}} \operatorname{tr}\left[\pi_{\omega_{0}, \bar{\phi}}(f)\left(\otimes_{k=1}^{n-1} Q_{k}\right)\right] d \bar{\phi},
$$

where $Q_{k}=\otimes_{i=1}^{n-k} \pi_{i}\left(t_{i, i+1}^{*} t_{i, i+1}\right)^{i}\left(1-q^{2 i}\right)^{-1}, T_{0}$ is the greatest torus in $U_{q}(n)$.

The formula for the invariant integral on $S_{q}^{n, m}$ is:

$$
\nu_{n, n-m}(f)=(2 \pi)^{-m} \int_{T_{m}} \operatorname{tr}\left[\pi_{\omega_{m}, \bar{\phi}}(f)\left(\otimes_{k=1}^{m-1} Q_{k}\right)\right] d \bar{\phi},
$$

where $Q_{k}$ are the same as above, $T_{m}$ is the greatest torus in $S_{q}^{n, m}$.

Proof. The induction step is given by the following equality:

$$
\nu_{n}=\left(\nu_{n, n-1} \otimes \nu_{n-1}\right)\left(\mathrm{id} \otimes \gamma_{1}\right) \Delta,
$$

where $\gamma_{1}: U_{q}(n) \rightarrow U_{q}(n-1)$ is an epimorphism, a map $P_{r}=\left(\mathrm{id} \otimes \nu_{n-1}\right)(\mathrm{id} \otimes$ $\left.\gamma_{1}\right) \Delta$ is exactly a projector from $U_{q}(n)$ on $S_{q}^{n, 1}$ such that $\left(\operatorname{id} \otimes P_{r}\right) \Delta=\Delta \circ P_{r}$ [4], [24], [25], [26]. In fact, by the properties of $P_{r}$ and $\nu_{n, n-1}$, one can see that the right-hand side of the above equality is exactly a right-invariant integral on $U_{q}(n)$. Since such an integral is unique (see 2.5), the above equality is true.

The base of the induction is the following expression for an invariant integral on $U_{q}(1): \nu_{1}(t)=0, \nu_{1}(1)=1$. Now, using the formula for $\nu_{n, n-1}$, we obtain the statements of the lemma.

Corollary 1. The invariant integrals $\nu_{n}$ and $\nu_{n, n-m}$ are the faithful states on the corresponding $C^{*}$-algebras $\mathbf{C}\left(U_{q}(n)\right)$ and $\mathbf{C}\left(S_{q}^{n, m}\right)$.

Remark 7. The statement of the above corollary was obtained in [13] by different considerations.

\section{2 .}

The construction of the invariant integral allows us to prove the following:

Theorem 5. For the series of the greatest representations $\pi_{\omega_{m}, \bar{\phi}}$ of $S_{q}^{n, m}$ the following statement holds:

$$
\cap_{\bar{\phi}} \operatorname{Ker} \pi_{\omega_{m}, \bar{\phi}}=0 .
$$


Proof. Let $\cap_{\bar{\phi}} \operatorname{Ker} \pi_{\omega_{m}, \bar{\phi}}=L \ni v$. Since $L$ is a $*$-ideal, then $v v^{*} \in L$, and we get $\pi_{\omega_{m}, \bar{\phi}}\left(v v^{*}\right)=0$. But at the same time, $v v^{*}$ is a strictly positive element, hence $\nu_{n, n-m}\left(v v^{*}\right)>0$. This contradiction proves the theorem.

The above theorem allows us to state that the representation

$$
R_{n}(f)=\oplus \int_{T_{m}} \pi_{\omega_{m}, \bar{\phi}}(f) d \bar{\phi}
$$

is the faithful representation of $C^{*}$ algebra $\mathbf{C}\left(S_{q}^{n, m}\right)$.

This result generalizes the construction of the faithful representation for $S_{q}^{n, 1}[\mathbf{2 8}]$.

6. Double cosets $U_{q}(n-m) \backslash U_{q}(n) / U_{q}(n-m)$.

6.1 .

The definition of $U_{q}(n-m) \backslash U_{q}(n) / U_{q}(n-m)$ as the intersection of $U_{q}(n-$ $m) \backslash U_{q}(n)$ and $U_{q}(n) / U_{q}(n-m)$ along with the results of Section 3 allow to describe the hypergroup structure on it more precisely than in Section 2.7. First, a straightforward corollary of Subsection 3.3 and Theorem 1 is given by the following:

Lemma 3. The algebra $U_{q}(n-m) \backslash U_{q}(n) / U_{q}(n-m)$ is generated by the generators $t_{i j}, t_{i j}^{*}(n-m+1 \leq i, j \leq n)$ for which the relations of commutation (17), (18), (19) hold.

Moreover, this algebra has the following comodule decomposition:

$$
U_{q}(n-m) \backslash U_{q}(n) / U_{q}(n-m) \sim \oplus_{\lambda} V^{L}(\lambda)_{n-m}^{0} \otimes V^{R}(\lambda)_{n-m}^{0},
$$

where $\lambda$ is an integral dominant weight such that $\mu^{n-m}=0 \prec \lambda$.

Remark 8. We can also consider an algebra $U_{q}\left(n-m_{1}\right) \backslash U_{q}(n) / U_{q}\left(n-m_{2}\right)$. This algebra is generated by generators $t_{i j}, t_{i j}^{*}\left(n-m_{1}+1 \leq i \leq n, n-m_{2}+\right.$ $1 \leq j \leq n$ ) for which the relations of commutation (17), (18), (19) hold.

\section{2 .}

One can introduce a new coproduct on the algebra $U_{q}(n-m) \backslash U_{q}(n) / U_{q}(n$ $-m): \quad \tilde{\Delta}:=\left(\mathrm{id} \otimes \nu_{n-m} \otimes \mathrm{id}\right)\left(\mathrm{id} \otimes \gamma_{m} \otimes \mathrm{id}\right)(\Delta \otimes \mathrm{id}) \Delta$, where $\nu_{n-m}$ is the invariant integral on $U_{q}(n-m), \gamma_{m}$ is the epimorphism from $U_{q}(n)$ to $U_{q}(n-$ $m$ ) and $\Delta$ is the coproduct on $U_{q}(n)$.

Lemma 4. The coproduct $\tilde{\Delta}$ "respects" the comodule structure of $U_{q}(n-$ $m) \backslash U_{q}(n) / U_{q}(n-m)=\oplus_{\lambda} U_{q}(n-m) \backslash W(\lambda) / U_{q}(n-m):$

$$
\begin{gathered}
\tilde{\Delta}: U_{q}(n-m) \backslash W(\lambda) / U_{q}(n-m) \rightarrow \\
U_{q}(n-m) \backslash W(\lambda) / U_{q}(n-m) \otimes U_{q}(n-m) \backslash W(\lambda) / U_{q}(n-m) .
\end{gathered}
$$


Proof. The coproduct $\Delta$ acts on $W(\lambda) / U_{q}(n-m)$ in the following way: $\Delta: W(\lambda) / U_{q}(n-m) \rightarrow W(\lambda) \otimes W(\lambda) / U_{q}(n-m)$ and hence $(\Delta \otimes \mathrm{id}) \Delta:$ $U_{q}(n-m) \backslash W(\lambda) / U_{q}(n-m) \rightarrow U_{q}(n-m) \backslash W(\lambda) \otimes W(\lambda) \otimes W(\lambda) / U_{q}(n-m)$. It was shown earlier that $W(\lambda)$ can be decomposed into the direct sum as the right $U_{q}(n-m)$-comodule: $W(\lambda)=\oplus_{\mu^{n-m} \prec \lambda} W\left(\lambda, \mu^{n-m}\right)$.

In the similar way $W(\lambda)$ can be decomposed as the left $U_{q}(n-m)$-comodule: $W(\lambda)=\oplus_{\mu^{n-m} \prec \lambda} W\left(\mu^{n-m}, \lambda\right)$.

Then one has: $\left(\mathrm{id} \otimes \gamma_{m} \otimes \mathrm{id}\right)(\Delta \otimes \mathrm{id}) \Delta: U_{q}(n-m) \backslash W(\lambda) / U_{q}(n-m) \rightarrow$ $\oplus_{\mu^{n-m} \prec \lambda} U_{q}(n-m) \backslash W\left(\lambda, \mu^{n-m}\right) \otimes W\left(\mu^{n-m}\right) \otimes W\left(\mu^{n-m}, \lambda\right) / U_{q}(n-m)$. One uses the fact that $\nu_{n-m}\left(W\left(\mu^{n-m}\right)\right)=0$ if $\mu^{n-m} \neq 0$ and $W(0)=\mathbf{C}$; $W(\lambda) / U_{q}(n-m)=W(\lambda, 0) ; U_{q}(n-m) \backslash W(\lambda)=W(0, \lambda)$.

After that $\left(\mathrm{id} \otimes \nu_{n-m} \gamma_{m} \otimes \mathrm{id}\right)(\Delta \otimes \mathrm{id}) \Delta: U_{q}(n-m) \backslash W(\lambda) / U_{q}(n-m) \rightarrow$ $U_{q}(n-m) \backslash W(\lambda, 0) \otimes W(0) \otimes W(0, \lambda) / U_{q}(n-m)=U_{q}(n-m) \backslash W(\lambda) / U_{q}(n-$ $m) \otimes U_{q}(n-m) \backslash W(\lambda) / U_{q}(n-m)$.

\section{3.}

Theorem 2 allows to get the following statement referring to representations of double cosets:

Lemma 5. (i) Representations $\pi_{j_{1}} \otimes \cdots \otimes \pi_{j_{l}}$ corresponding to the elements $\omega$ of the least possible length (among the representatives of the class from $\left.S_{n-m} \backslash S_{n} / S_{n-m}\right)$ are irreducible representations of the double cosets $U_{q}(n-$ $m) \backslash U_{q}(n) / U_{q}(n-m)$.

(ii) The series of greatest representations of $U_{q}(n-m) \backslash U_{q}(n) / U_{q}(n-m)$ $\pi_{m \omega_{m}, \bar{\phi}}=\pi_{n-m} \otimes \pi_{n-1} \otimes \pi_{n-m-1} \otimes \cdots \otimes \pi_{n-2} \otimes \cdots \otimes \pi_{n-2 m} \otimes \cdots \otimes \pi_{n-m} \otimes \kappa_{\bar{\phi}}$ is parametrized by the greatest torus in $U_{q}(n-m) \backslash U_{q}(n) / U_{q}(n-m)$. Here ${ }_{m} \omega_{m}$ are the greatest elements of double cosets $S_{n-m} \backslash S_{n} / S_{n-m}$.

(iii) The representation $R_{n}(f)=\oplus \int_{T_{m}} \pi_{\omega_{m}, \bar{\phi}}(f) d \bar{\phi}$ is the faithful representation of $C^{*}$ algebra generated by $U_{q}(n-m) \backslash U_{q}(n) / U_{q}(n-m)$.

Finally, Lemma 2 gives a formula for the invariant integral on $U_{q}(n-$ $m) \backslash U_{q}(n) / U_{q}(n-m)$ which is the restriction of $\nu_{n, n-m}$ on this algebra. The mentioned formula shows that the invariant integral is the faithful state on the $C^{*}$-algebra generated by the double cosets.

\section{References}

[1] E. Abe, Hopf algebras, Cambridge Tracts in Math., Vol. 74, Cambridge University Press, 1980.

[2] N. Bourbaki, Elements de mathématique, Algèbre, Chapitre 2, Hermann, Paris, 1962.

[3] K. Bragiel, On the spherical and zonal spherical functions on a compact quantum group, Letters in Math. Phys., 22 (1991), 195-201.

[4] Y. Chapovsky and L. Vainerman, Hypergroup structures, associated with a pair of quantum groups $\left(S U_{q}(n), U_{q}(n-1)\right)$, in 'Methods of functional analysis in problems of mathematical physics', pages 47-69, Inst. Math. Acad. Sci. Ukraine, Kiev, 1992. 
[5] V.G. Drinfeld, Quantum Groups, in 'Proceedings of the International Congress of Mathematicians', Berkeley, California, USA, (1986), 798-820.

[6] J. Faraut, Analyse harmonique sur les paires de Guelfand et les espaces hyperboliques, in 'Analyse Harmonique', pages 315-446, CIMPA, Nice, 1982.

[7] P. Floris, A Non-Commutative Discrete Hypergroup Associated with q-Disk Polynomials, preprint, 1994.

[8] S. Gelbart, A theory of Stiefel harmonics, Trans. Amer. Math. Soc., 192 (1974), 29-50.

[9] M. Jimbo, A q-difference analogue of $u(g)$ and the Yang-Baxter equation, Lett. Math. Phys., 10 (1985), 63-69.

[10] T. Koornwinder, Orthogonal polynomials in connection with quantum groups, in 'Orthogonal Polynomials: Theory and Practice' (P. Nevai, editor), Vol. 294, (1990), 257-292, Norwell, MA, NATO-ASI Series C, Kluwer.

[11] _ Positive convolution structures associated with quantum groups, in 'Probability Measures on Groups', X, pages 249-268, Plenum, 1991.

[12] _ Discrete hypergroups associated with compact quantum Gel'fand pairs, Contemporary Math., 183 (1995), 213-235.

[13] G. Nagy, A Framework for Deformation Quantization, Ph. D. Thesis, Berkeley, 1992.

[14] M. Noumi, H. Yamada and K. Mimachi, Finite dimensional representations of the quantum group $G L_{q}(n ; C)$ and the zonal spherical functions on $U_{q}(n-1) \backslash U_{q}(n)$, Japan. J. Math., 19(1) (1993), 31-80.

[15] G. Podkolzin, Infinitesimal algebra for hypercomplex system with a basis $S O(4) / / S O(2)$, Ukrainian Math. Journal, 42(3) (1990), 427-429; English transl. in Ukrainian Math. J., 3 (1990), 98-101.

[16] _ Infinitesimal object for hypercomplex system generated by double cosets, and nonlinear differential equations, Funktsional. Anali. Prilozhen., 25(1) (1991), 91-93; English transl. in Funct. Anal. Appl., 25 (1991), 78-80.

[17] P. Podles, Quantum spheres, Lett. Math. Phys., 14 (1987), 193-202.

[18] N. Reshetikhin, L. Takhtadjan and L. Faddeev, Quantization of Lie groups and Lie algebras, Algebra i Analiz, 1(1) (1989), 178-206. English transl. in Leningrad Math. J., 1 (1990), 193-225.

[19] Y. Soibelman, Irreducible representations of the algebra of functions on the Quantum group $S U(n)$ and Shubert cells, Dokl. Acad. Nauk SSSR, 307 (1989), 41-45; English Transl. in Soviet Math. Dokl., 40 (1990).

[20] The algebra of functions on a compact Quantum group and its representations, Algebra i Analiz, 2(1) (1990), 190-211; English Transl. in Leningrad Math. J., 2(1) (1991), 161-178.

[21] Y. Soibelman and L. Vaksman, On some problems in the Theory of Quantum groups, Adv. in Soviet Math., 9 (1992), 3-55.

[22] R. Strichartz, The explicit fourier decomposition of $L^{2}(S O(n) / S O(n-m))$, Canadian Math. Journal, 27 (1975), 294-310.

[23] L. Vainerman, Duality for algebras with involution and generalized shift operators, Journal of Soviet Math., 42(6) (1988), 52-59.

[24] _ Gel'fand pairs of quantum groups, hypergroups and q-special functions, Contemporary Mathematics, 183 (1995), 373-394. 
$[25]$ Hypergroup structures associated with Gel'fand pairs of compact quantum group, Asterisque, 232 (1995), 231-243.

[26] L. Vainerman and Y. Weiss, Hypergroup associated with double cosets of the quantum group $S U_{q}(2)$, in 'Applications of the functional analysis methods in math. physics', pages 52-59, Inst. Math. Acad. Sci. Ukraine, Kiev, 1991, Russian; to be transl. in Selecta Math. Sov.

[27] L. Vaksman and Y. Soibelman, Algebra of functions on the quantum group SU(2), Funct. Analyz i ego Pril., 22(3) (1988), 1-14; English transl. Funct. Anal. Appl., 22 (1988).

[28] _ Algebra of functions on quantum group $S U(n+1)$ and odd dimensional spheres, Algebra i Analyz, 2(5) (1990), 101-120; English transl. in Leningrad Math. J., 2(5) (1991), 1023-1042.

[29] S. Woronowicz, Compact matrix pseudogroups, Commun. Math. Phys., 111 (1987), 613-665.

Received November 6, 1996 and revised January 10, 1997. This research was supported in part by Ukrainian Foundation for Fundamental Studies and by International Science Foundation.

Kiev Technical University

KIEV 252033

UKRAINE

E-mail address: mathkiev@imat.gluk.apc.org

INTERNATIONAL SOLOMON UNIVERSITY

KIEV 252187

UKRAINE

E-mail address: wain@agrosys.kiev.ua

URA CNRS 747 (CASE 191)

Universite Paris VI

4 Pl. Jussieu, 75252

Paris Cedex 05

FRANCE 